\title{
Pitavastatin approved for treatment of primary hypercholesterolemia and combined dyslipidemia
}

\author{
This article was published in the following Dove Press journal: \\ Vascular Health and Risk Management \\ 29 October 2010 \\ Number of times this article has been viewed
}

\author{
Jun Sasaki \\ Pharmaceutical Medicine, \\ International University of Health \\ and Welfare Graduate School, \\ Fukuoka, Japan
}

\begin{abstract}
Pitavastatin was first developed in Japan and is expanding the regions in which it is clinically available. A considerable number of clinical studies have been conducted and published to date on the usefulness of pitavastatin for patients with primary hypercholesterolemia or combined dyslipidemia. Pitavastatin demonstrates potent low-density lipoprotein cholesterol reduction at low doses of 1-4 mg/day. It also affects the regression of coronary plaques, as observed in intravascular ultrasound-guided percutaneous coronary intervention studies. Moreover, the persistent, long-term high-density lipoprotein cholesterol elevation observed in the populations treated with pitavastatin is worthy of further attention. The reported improvements in lipid profiles are consistent among the studies conducted in Japan, Korea, Thailand, and Europe. In light of accumulating clinical experience worldwide, pitavastatin is now expected to establish its position for preventing and treating cardiovascular disease.
\end{abstract}

Keywords: randomized clinical trial, Japan, Korea, Thailand, Europe

\section{Introduction}

Statins have contributed greatly to medical care since the late twentieth century through their role in the primary and secondary prevention of cardiovascular disease and reduction in total mortality. Compactin, the first statin, was discovered in the mid1970s by Akira Endo, who had been exploring a substance from fungi that inhibited 3-hydroxy-3-methylglutaryl-coenzyme A (HMG-CoA) reductase, a rate-determining enzyme in the cholesterol synthesis pathway. ${ }^{1}$ Thereafter, lovastatin, pravastatin, and simvastatin became clinically available in 1987, 1989, and 1992, respectively. Moreover, large-scale clinical studies, including the West of Scotland Coronary Prevention Study, ${ }^{2}$ the Air Force/Texas Coronary Atherosclerosis Prevention Study, ${ }^{3}$ Cholesterol and Recurrent Events, ${ }^{4}$ and Scandinavian Simvastatin Survival Study ${ }^{5}$ were successfully conducted, leading to the era of statin therapy. Thereafter, fluvastatin, cerivastatin (withdrawn from the worldwide market in 2001), atorvastatin, pitavastatin, and rosuvastatin were developed.

Aggressive lowering of low-density lipoprotein cholesterol (LDL-C) levels is thought to be associated with a greater risk reduction for cardiovascular disease. Hence, aggressive lowering of LDL-C levels may benefit patients believed to be at risk for cardiovascular disease, even if their LDL-C levels are within the normal range for the general population, as well as patients with high LDL-C levels. ${ }^{6}$ Statins developed more recently exert more potent LDL-C reduction, allowing for more aggressive LDL-C-lowering therapy.
Correspondence: Jun Sasaki

I-3-I Nagahama, Chuo-ku,

Fukuoka, 810-0072 Japan

$\mathrm{Tel}+81927|||| \mid 26$

Fax +8I 927391609

Email jsas@nifty.com 
The withdrawal of cerivastatin was due to reports that the risk for fatal rhabdomyolysis might be increased when it was used concomitantly with gemfibrozil. ${ }^{7}$ Since this incident, drug-drug interactions have attracted attention. Pitavastatin is the only statin (including its lactone form) that is scarcely metabolized by cytochrome P450 (CYP), a drug-metabolizing enzyme in the liver. ${ }^{8}$ Hence, pitavastatin is thought to be minimally involved in drug-drug interactions mediated by CYP. This is an important property of pitavastatin because a lot of drug-drug interactions are recognized as a result of interferences in the metabolic pathway, the majority of which involve CYP isoenzymes. In fact, physicians should always take potential drug interactions into account with any drug to be administered, because multiple medications are often needed for patients with elevated cholesterol. As with other statins, other major causes of drug-drug interactions, ie, drug transporters, such as organic anion transporting polypeptide, also need to be carefully considered for pitavastatin. ${ }^{9}$

Pitavastatin was first developed and launched in Japan in 2003. Its safety and efficacy in Japan was established by the large-scale, prospective, post-marketing LIVES (LIValo Effectiveness and Safety) study, conducted in 20,000 patients with a two-year follow-up for each patient over six years since $2003 .{ }^{10}$ Pitavastatin's usefulness has been reported in the results of many other clinical studies. To date, the findings indicate that pitavastatin has not only a potent LDL-Clowering effect but also a long-term high-density lipoprotein cholesterol (HDL-C)-elevating effect. ${ }^{11}$ Pitavastatin became available in Korea in 2005, followed by approval in Thailand and China. It was recently approved by the Food and Drug Administration (FDA) in the US in August 2009. Pitavastatin is also being reviewed in Europe, and is expected to be approved there in 2010. Therefore, pitavastatin's usefulness is expected to be confirmed worldwide in the future. This paper reviews the clinical studies conducted and published to date on the usefulness of pitavastatin for patients with primary hypercholesterolemia and those with combined dyslipidemia, for which pitavastatin is indicated.

\section{Methods}

Articles for this review were selected to examine LDL-C reduction by dose, safety, and the long-term HDL-Celevating effect of pitavastatin in patients with primary hypercholesterolemia or combined dyslipidemia.

To examine LDL-C reduction and safety of pitavastatin at different doses, a systematic review was conducted on randomized active-controlled studies that compared the effects of pitavastatin with those of other statins in patients with primary hypercholesterolemia or combined dyslipidemia. Relevant articles were searched for in PubMed on 26 April 2010, using the search term "pitavastatin" and "randomized". Thirty-seven papers ${ }^{11-47}$ were found. Four of these papers were reviews, ${ }^{11-14}$ two were articles on rationale and design of clinical studies, ${ }^{15,16}$ two were nonclinical studies, ${ }^{17,18}$ and five were pharmacokinetic studies, ${ }^{19-23}$ so were excluded. One interventional study of an unspecified statin, ${ }^{24}$ two clinical studies using unspecified doses of pitavastatin, ${ }^{25,26}$ and three studies that did not report percent change in LDL-C with different doses of pivastatin ${ }^{27-29}$ were also excluded. In addition, one nonrandomized comparative study and seven studies that compared pitavastatin treatment with nonstatin treatment, lifestyle modification, or combined treatment with pitavastatin and other agents were further excluded, ${ }^{30-36}$ as were two studies conducted in patients with familial hypercholesterolemia. ${ }^{37,38}$ However, one study conducted in patients who had glucose intolerance and baseline lipid levels similar to those in the other included articles ${ }^{39}$ and another two studies conducted in patients with acute coronary syndrome $(\mathrm{ACS})^{40,41}$ were included. Moreover, two Phase III studies reported in Europe that were not found from the search results (one found in a review article and the other reported in a congress meeting were included).$^{48,49}$ Thus, a total of 11 articles ${ }^{39-47}$ were reviewed.

Five studies of the long-term treatment with pitava$\operatorname{statin}^{39,48-53}$ were reviewed. These papers were selected in a similar way to the abovementioned clinical studies, and all had study durations of more than 52 weeks or 12 months. Of those identified in PubMed using the keyword "pitavastatin", review articles, commentaries, nonclinical and pharmacokinetic studies, and those focusing on rationale and/or study were excluded. Articles on HDL-C were also excluded if patients were treated with unspecified statins, data for HDL-C levels were not available for more than three visits, or the paper was a case report for one or two patients. A further study not identified on PubMed, but in which one of the authors of the present review participated, was included. ${ }^{50}$

\section{LDL-C reduction and safety of pitavastatin}

Randomized controlled studies that compared the effects of pitavastatin with those of other statins in patients with primary hypercholesterolemia or combined dyslipidemia, including some patients with glucose intolerance or ACS, were reviewed (Table 1). Relatively high percent reductions in LDL-C were reported, including $37.4 \%$ using $1 \mathrm{mg}$ /day pitavastatin in Thailand, ${ }^{42} 42.6 \%$ using $2 \mathrm{mg}$ /day pitavastatin 


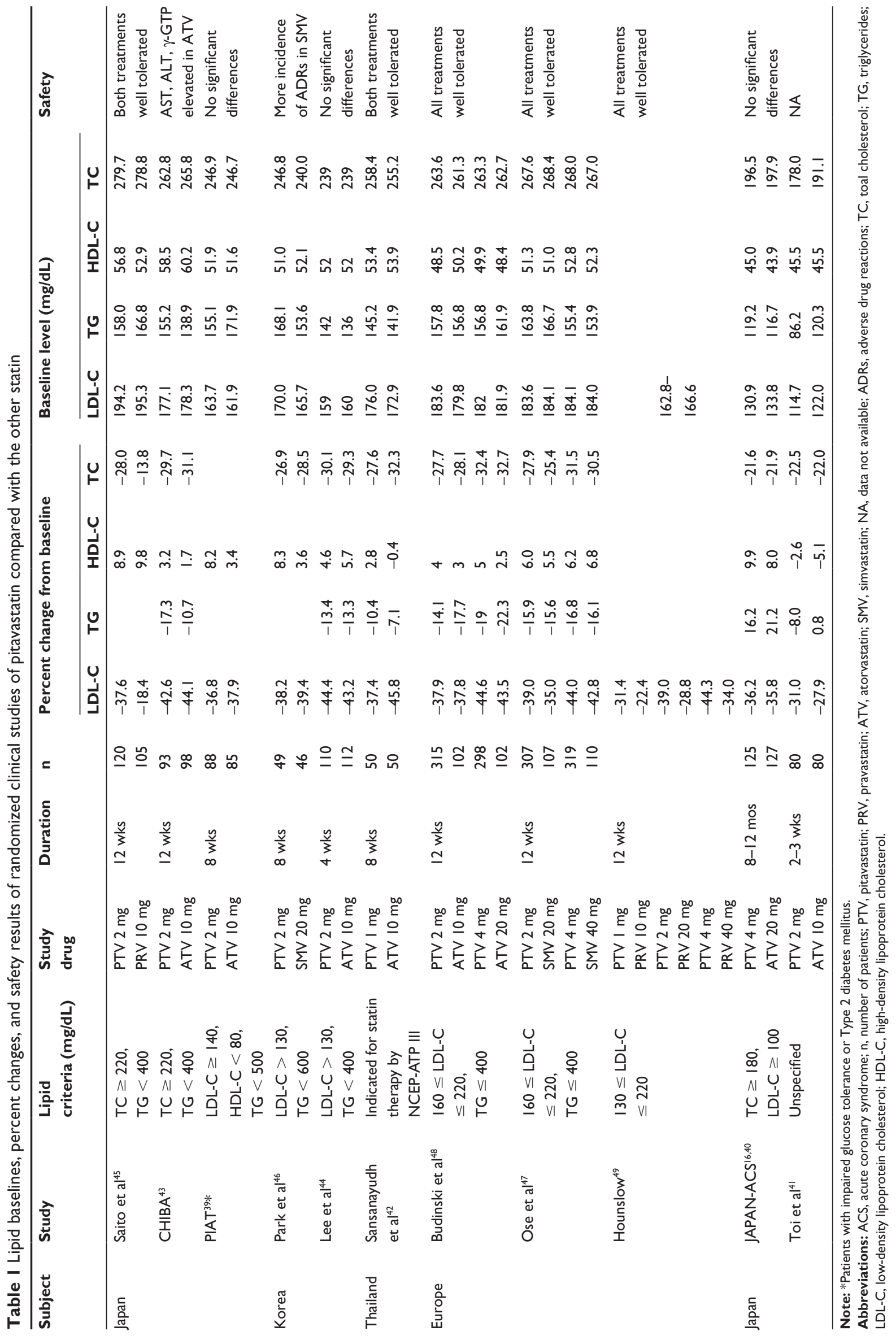


in CHIBA (Collaborative Study on Hypercholesterolemia Drug Intervention and their Benefits for Atherosclerosis Prevention $)^{43}$ conducted in Japan, and 44.4\% using $2 \mathrm{mg} /$ day pitavastatin in a Korean study that used atorvastatin as a control, ${ }^{44}$ respectively. The LDL-C-lowering effects of $2 \mathrm{mg} /$ day pitavastatin in other studies conducted in Japan, Korea, and Europe ${ }^{39,43-49}$ ranged from $36.8 \%$ to $39 \%$. The LDL-Clowering effects of $4 \mathrm{mg}$ /day pitavastatin ranged from $44.0 \%$ to $44.6 \%$ in the Phase III studies conducted in Europe. ${ }^{47-49}$ The LDL-C-lowering effects of $1 \mathrm{mg}$ /day pitavastatin was $31.4 \%$ in a Phase III study conducted in Europe. ${ }^{49}$ Randomized controlled studies that compared the LDL-C-lowering effect of $4 \mathrm{mg} /$ day pitavastatin with that of other statins in patients with primary hypercholesterolemia or combined dyslipidemia have not been reported in Japan, Korea, or Thailand. However, two studies conducted in patients with ACS in Japan showed that LDL-C levels were reduced by $36 \%$ from $131 \mathrm{mg} / \mathrm{dL}$ at baseline and by $31 \%$ from $115 \mathrm{mg} / \mathrm{dL}$ at baseline. ${ }^{40,41}$

The LDL-C-lowering effects following the administration of $2 \mathrm{mg} /$ day and $4 \mathrm{mg}$ /day pitavastatin are thought to be comparable with those observed following the administration of $10 \mathrm{mg} /$ day and $20 \mathrm{mg} /$ day atorvastatin and $20 \mathrm{mg} /$ day and $40 \mathrm{mg} /$ day simvastatin. The safety and tolerability of pitavastatin were similar to those of controls in the randomized controlled studies. Each study is outlined below.

\section{Phase III Japanese study of pitavastatin versus pravastatin}

The effects of $2 \mathrm{mg} /$ day pitavastatin were compared with the effects of a regular dose of $10 \mathrm{mg} /$ day pravastatin, which was the most commonly used statin in Japan at the time of this study. ${ }^{45}$ In 225 patients with total cholesterol (TC) levels $\geq$ $220 \mathrm{mg} / \mathrm{dL}$ and triglyceride (TG) levels $<400 \mathrm{mg} / \mathrm{dL}$, the LDL-C-lowering effect of pitavastatin after administration for 12 weeks was significantly better than that of pravastatin $(37.6 \%, 95 \%$ confidence interval [CI] 35.3-39.9 versus $18.4 \%, 95 \%$ CI $16.1-20.7$, respectively; the $95 \%$ CI of the difference was 11.8-16.5). In patients with baseline TG levels $\geq 150 \mathrm{mg} / \mathrm{dL}$, the TG-lowering-effect was $23.3 \%$ (95\% CI 13.7-32.9) and 20.2\% (95\% CI 12.0-28.4) in the pitavastatin group and the pravastatin group, respectively. HDL-C levels increased by $8.9 \%$ (95\% CI 6.4-11.4) and $9.8 \%$ (95\% CI 6.4-11.4), respectively.

\section{CHIBA study}

Since pitavastatin was launched in Japan in 2003, several investigator-initiated randomized comparative studies have been conducted, including the Chiba trial. ${ }^{43}$ In this trial, 251 hypercholesterolemic patients with TC levels $\geq 220 \mathrm{mg} / \mathrm{dL}$ and TG levels $<400 \mathrm{mg} / \mathrm{dL}$ were randomized to receive $2 \mathrm{mg} /$ day pitavastatin or $10 \mathrm{mg} /$ day atorvastatin. The primary endpoint was the percent change in nonHDL-C levels from baseline after 12 weeks of treatment. Non-HDL-C levels were reduced by $39 \%(P<0.001)$ in the pitavastatin group and by $40.3 \%(P<0.001)$ in the atorvastatin group. The percent reduction in non-HDL-C levels showed a statistically significant positive correlation with waist circumference and body mass index (BMI) only in the atorvastatin group $(\mathrm{r}=0.33, P=0.034$ and $\mathrm{r}=0.279$, $P=0.022$, respectively); no significant correlation for these parameters was found in the pitavastatin group. There were no statistically significant between-group differences in the percent changes in LDL-C, TG, HDL-C, and non-HDL-C. HDL-C increased significantly only in the pitavastatin group (3.2\%, $P=0.033)$. The percent changes in LDL-C were $42.6 \%(P<0.001)$ and $44.1 \%(P<0.001)$ in the pitavastatin and atorvastatin groups, respectively. Subgroup analysis was also conducted in patients with metabolic syndrome. In this subanalysis, LDL-C reduction in the pitavastatin group showed a tendency to be significantly superior to that in the atorvastatin group $(P=0.050)$, and the percent change in TG $(-25.2 \%, P<0.001)$ as well as HDL-C (6.7\%, $P=0.019)$ was statistically significant only in the pitavastatin group. Both treatments were well tolerated, but aspartate aminotransferase, alanine aminotransferase, and gamma-glutamyl transpeptidase became elevated in atorvastatin group.

\section{PIAT study}

The PIAT study was conducted to examine the effects of pitavastatin and atorvastatin on HDL-C levels in patients with elevated LDL-C and glucose intolerance. ${ }^{39}$ In this study, the primary endpoint was the percent change in HDL-C levels following treatment with $2 \mathrm{mg}$ /day pitavastatin or $10 \mathrm{mg}$ /day atorvastatin for 52 weeks (described further on in this review with regard to the HDL-C-elevating effect of pitavastatin). The subjects were 173 hypercholesterolemic patients with glucose intolerance or Type 2 diabetes mellitus who had LDL-C levels $\geq 140 \mathrm{mg} / \mathrm{dL}$, HDL-C levels $<80 \mathrm{mg} / \mathrm{dL}$, and TG levels $<500 \mathrm{mg} / \mathrm{dL}$. The LDL-C reductions in the pitavastatin group and atorvastatin group after treatment for eight weeks (a treatment duration similar to that used in other studies) were $36.8 \%$ and $37.9 \%$, respectively $(P=0.61)$, which were reported by Sasaki in the XVI International Symposium on Drugs Affecting Lipid Metabolism in New York, USA in 2007. No significant differences between 
the two groups were observed with regard to worsening of glucose metabolism or incidence of adverse events.

\section{Comparative study of pitavastatin and simvastatin in Korea}

In this study, 103 hypercholesterolemic patients with LDL-C levels $\geq 130 \mathrm{mg} / \mathrm{dL}$ and TG levels $<600 \mathrm{mg} / \mathrm{dL}$ received $2 \mathrm{mg} /$ day pitavastatin or $20 \mathrm{mg} /$ day simvastatin for eight weeks. ${ }^{46}$ The LDL-C reduction was $38.2 \%$ in the pitavastatin group and $39.4 \%$ in the simvastatin group; there was no significant difference between the groups $(P=0.648)$ for percent changes in TC, TG, and HDL-C, or the proportion of patients attaining the LDL-C treatment goals recommended in the National Cholesterol Education Program (NCEP)Adult Treatment Panel (ATP) III guidelines. The incidence of adverse events was not significantly different between the groups $(25.0 \%$ in the pitavastatin group and $37.3 \%$ in the simvastatin group, $P=0.179$ ). However, the incidence of adverse events that had an undeniable causal relationship with the study drug was $15.4 \%$ in the pitavastatin group and $37.3 \%$ in the simvastatin group, indicating a significant difference between the groups $(P=0.015)$.

\section{Comparative study of pitavastatin and atorvastatin in Korea}

In this study, 268 hypercholesterolemic patients with LDL-C levels $\geq 130 \mathrm{mg} / \mathrm{dL}$ and TG levels $<400 \mathrm{mg} / \mathrm{dL}$ received $2 \mathrm{mg} /$ day pitavastatin or $10 \mathrm{mg} /$ day atorvastatin for four weeks, followed another four-week treatment with uptitration when a patient did not achieve the NCEP-ATP III goal. ${ }^{44}$ Thus, the study drugs were administered for a total of eight weeks. The LDL-C reduction was $44.4 \%$ in the pitavastatin group and $43.2 \%$ in the atorvastatin group after four weeks, indicating no significant difference between the groups $(P=0.41)$. Tolerability was good in both groups. The incidence of adverse events was $19.1 \%$ in the pitavastatin group and $25.0 \%$ in the atorvastatin group. Increased creatine kinase levels equal to or higher than 10 times the upper limit of the normal range, with muscle symptoms, did not occur.

\section{Comparative study of pitavastatin and atorvastatin in Thailand}

A study conducted in Thailand compared the LDL-C reduction using $1 \mathrm{mg} /$ day pitavastatin with that of $10 \mathrm{mg} /$ day atorvastatin. ${ }^{42}$ In a total of 100 patients with hypercholesterolemia, the LDL-C reduction after treatment $(P<0.001)$ in $74 \%$ and $84 \%$ of subjects in the pitavastatin and atorvastatin groups, respectively, achieved the LDL-C goal recommended in the NCEP-ATP III guidelines, with no significant difference between the groups $(P=0.220)$. Tolerability was good in both groups. Elevation of alanine transaminase levels equal to or higher than three times the upper limit of normal or elevation of creatine kinase levels equal to or higher than 10 times the upper limit of normal did not occur. The monthly cost per percent LDL-C reduction by pitavastatin $1 \mathrm{mg} /$ day was less than that for atorvastatin $10 \mathrm{mg} /$ day in Thailand.

\section{Comparative study of pitavastatin and atorvastatin in Europe}

This study was conducted in 821 patients with hypercholesterolemia or combined dyslipidemia whose LDL-C levels were between 160 and $220 \mathrm{mg} / \mathrm{dL}$ and whose TG levels were $\leq 400 \mathrm{mg} / \mathrm{dL}$. ${ }^{48}$ The study aimed to compare the LDL-C reductions following treatment with pitavastatin for 12 weeks with those after atorvastatin treatment for the same duration (pitavastatin $2 \mathrm{mg} /$ day versus atorvastatin $10 \mathrm{mg}$ /day and pitavastatin $4 \mathrm{mg} /$ day versus atorvastatin $20 \mathrm{mg} /$ day). The LDL-C reductions were $37.9 \%$ (pitavastatin $2 \mathrm{mg} /$ day), $37.8 \%$ (atorvastatin $10 \mathrm{mg} /$ day), $44.6 \%$ (pitavastatin $4 \mathrm{mg} /$ day), and $43.5 \%$ (atorvastatin $20 \mathrm{mg} /$ day), indicating no significant differences between the pitavastatin and atorvastatin groups ( $P=0.93$ between the lower dose groups and 0.57 between the higher dose groups, respectively). There were no significant between-group differences in the percent changes in TC, TG, HDL-C, and non-HDL-C or the rates of achievement of the LDL-C goal. Tolerability was good in both groups. The incidence of adverse events was $19.0 \%$ (pitavastatin $2 \mathrm{mg} /$ day), $16.7 \%$ (atorvastatin $10 \mathrm{mg} /$ day), $16.7 \%$ (pitavastatin $4 \mathrm{mg} /$ day), and $22.3 \%$ (atorvastatin $20 \mathrm{mg} /$ day).

\section{Comparative study of pitavastatin and simvastatin in Europe}

This study was conducted in 857 patients with hypercholesterolemia or combined dyslipidemia whose LDL-C levels were between 160 and $220 \mathrm{mg} / \mathrm{dL}$ and whose TG levels were $\leq 400 \mathrm{mg} / \mathrm{dL} .{ }^{47}$ The study compared the LDL-C reductions following treatment with pitavastatin for 12 weeks with those after treatment with simvastatin for the same duration (pitavastatin $2 \mathrm{mg} /$ day versus simvastatin $20 \mathrm{mg} /$ day and pitavastatin $4 \mathrm{mg}$ /day versus simvastatin $40 \mathrm{mg}$ /day). The LDL-C reductions were $39.0 \%$ (pitavastatin $2 \mathrm{mg} /$ day), 35.0\% (simvastatin $20 \mathrm{mg} /$ day), $44.0 \%$ (pitavastatin $4 \mathrm{mg} /$ day), and $42.8 \%$ (simvastatin $40 \mathrm{mg} /$ day). The LDL-C reduction following treatment with $2 \mathrm{mg}$ /day pitavastatin was significantly better than that 
after treatment with $20 \mathrm{mg}$ /day simvastatin $(P=0.014)$. The percent changes in TC and non-HDL-C levels and the rate of achievement of the LDL-C goal recommended in the European Atherosclerosis Society guidelines in the pitavastatin $2 \mathrm{mg} /$ day group were similarly superior to those in the simvastatin $20 \mathrm{mg} /$ day group $(P=0.041$, 0.021 , and 0.049 , respectively). The percent changes in TG and HDL-C and the rate of achievement of the LDL-C goal recommended in the NCEP-ATP III guidelines did not differ significantly between the treatment groups. Tolerability was good in both groups. The incidence of adverse events was $35.4 \%$ (pitavastatin $2 \mathrm{mg} /$ day), 33.6\% (simvastatin $20 \mathrm{mg} /$ day), $32.2 \%$ (pitavastatin $4 \mathrm{mg} /$ day), and $27.3 \%$ (simvastatin $40 \mathrm{mg} /$ day).

\section{Comparative study of pitavastatin and pravastatin in Europe}

This study was conducted in elderly patients with hypercholesterolemia or combined dyslipidemia whose LDL-C levels were between 130 and $220 \mathrm{mg} / \mathrm{dL}$ and whose TG levels were $\leq 400 \mathrm{mg} / \mathrm{dL}$. The study compared LDL-C reductions following administration of pitavastatin for 12 weeks with those following administration of pravastatin for the same duration (pitavastatin $1 \mathrm{mg} /$ day versus pravastatin $10 \mathrm{mg} /$ day, pitavastatin $2 \mathrm{mg}$ /day versus pravastatin $20 \mathrm{mg} /$ day, and pitavastatin $4 \mathrm{mg}$ /day versus pravastatin $40 \mathrm{mg} /$ day).$^{49} \mathrm{The}$ LDL-C reductions were $31.4 \%$ (pitavastatin $1 \mathrm{mg} /$ day), $22.4 \%$ (pravastatin $10 \mathrm{mg} /$ day), $39.0 \%$ (pitavastatin $2 \mathrm{mg} /$ day), $28.8 \%$ (pravastatin $20 \mathrm{mg} /$ day), $44.3 \%$ (pitavastatin $4 \mathrm{mg} /$ day), and $34.0 \%$ (pravastatin $40 \mathrm{mg} /$ day). The LDL-C reduction following pitavastatin treatment was significantly superior to that following pravastatin treatment at each dose comparison $(P<0.001)$. Adverse events were similar for the pitavastatin and pravastatin groups. Tolerability was good in both groups.

\section{JAPAN-ACS study}

JAPAN-ACS (Japanese Assessment of Pitavastatin and Atorvastatin in ACS) was conducted in 252 patients with ACS who had successfully undergone intravascular ultrasound-guided percutaneous coronary intervention. ${ }^{40} \mathrm{In}$ this study, the percent change in coronary plaque volume after treatment with $4 \mathrm{mg} /$ day pitavastatin for 8-12 months was compared with that after $20 \mathrm{mg}$ /day atorvastatin for the same duration. This study indicated the regressive effect of aggressive lipid-lowering therapy on coronary plaque volume in patients with ACS who had undergone intravascular ultrasound-guided percutaneous coronary intervention. The plaque regressive effect of pitavastatin was equivalent to that of atorvastatin. The LDL-C reduction was equivalent in the pitavastatin and atorvastatin groups (36.2\% and $35.8 \%$, respectively, $P=0.9$ ). The LDL-C reductions were relatively smaller than those seen in the other studies because the LDL-C levels were low at baseline $(130.9 \mathrm{mg} / \mathrm{dL}$ in the pitavastatin group and $133.8 \mathrm{mg} / \mathrm{dL}$ in the atorvastatin group). Unlike in other placebo-controlled studies, the percent change in LDL-C was not correlated with the percent change in plaque volume in the JAPANACS study. No significant differences were found in the incidence rate of cardiovascular or adverse events between the treatment groups.

\section{Pitavastatin versus atorvastatin using virtual histology intravascular ultrasound}

A study conducted in 160 patients with ACS who underwent percutaneous coronary intervention reported the early effects of 2-3 weeks' treatment with $2 \mathrm{mg}$ /day pitavastatin and $10 \mathrm{mg} /$ day atorvastatin on the components of coronary plaques. ${ }^{41}$ After treatment, the plaque volume index and fibrofatty plaque (a component of coronary plaques) volume index decreased significantly in the pitavastatin group $(P<0.05)$. The mean LDL-C level at baseline was $114.7 \mathrm{mg} / \mathrm{dL}$ in the pitavastatin group and $122.0 \mathrm{mg} / \mathrm{dL}$ in the atorvastatin group; both levels were lower than those in the JAPAN-ACS study. The LDL-C reduction was 31.0\% in the pitavastatin group $(P<0.001)$ and $27.9 \%$ in the atorvastatin group $(P<0.001)$. The percent change in plaque volume index showed a mild positive correlation with the percent change in LDL-C in the pitavastatin group. In a subgroup of patients in the pitavastatin group with a dense calcium plaque ratio less than $10 \%$, the percent change in fibrofatty volume index and the percent change in LDL-C correlated positively.

\section{HDL-C-elevating effect of pitavastatin}

HDL-C elevation following treatment with pitavastatin for 4-12 weeks ranged from $3.2 \%$ to $8.9 \%$ in a population with baseline HDL-C levels between 48.5 and $58.5 \mathrm{mg} / \mathrm{dL}$ in the randomized, active-controlled comparative study (see Table 1). The PIAT study ${ }^{39}$ compared treatment for 52 weeks with $2 \mathrm{mg} /$ day pitavastatin and $10 \mathrm{mg} /$ day atorvastatin. Because pitavastatin has an LDL-C-lowering effect similar to that of atorvastatin but is expected to have a superior HDL-C-elevating effect, PIAT also assessed the effects of 
pitavastatin and atorvastatin on the HDL-C levels of patients with elevated LDL-C and glucose intolerance. ${ }^{39}$ Patients with glucose intolerance or Type 2 diabetes mellitus were chosen because HDL-C levels, in addition to LDL-C levels, play a particularly important role in cardiovascular risk in these patients.

Pitavastatin was significantly superior to atorvastatin with regard to the increase in HDL-C levels after 52 weeks ( $8.8 \%$ versus $3.6 \%$, respectively, $P=0.034)$. This result was sustained throughout the 52 weeks of treatment. The percent change in the level of Apoprotein A-1, a major protein component of HDL-C, was significantly higher in the pitavastatin group than in the atorvastatin group after 52 weeks $(5.2 \%$ versus $1.1 \%$, respectively, $P=0.031$ ). Pitavastatin had a persistent HDL-C-elevating effect. Moreover, various nonrandomized studies that examined the effects of long-term administration of pitavastatin reported a sustained increase in HDL-C levels with pitavastatin treatment (see Figure 1). ${ }^{39,50-53}$ In addition to the potent LDL-C-lowering effect of pitavastatin, its long-term HDL-C-elevating effect is expected to provide some long-term benefits, but further confirmation of these results is warranted.

\section{Conclusion}

Pitavastatin has a potent serum LDL-C-lowering effect, even at low doses of $1-4 \mathrm{mg} /$ day. Pitavastatin also affects regression of coronary plaques, as observed in intravascular ultrasound-guided percutaneous coronary intervention studies. Moreover, the persistent long-term HDL-C elevation observed in patients treated with pitavastatin is worthy of further attention. The reported improvements in the lipid profile are consistent between the studies conducted in Japan, Korea, Thailand, and Europe. Pitavastatin has a lower propensity for drug-drug interactions via CYP, can exert a potent LDL-Clowering effect at relatively low doses, and has a favorable effect on coronary plaques. Therefore, it is expected that, as clinical experience of pitavastatin accumulates worldwide,

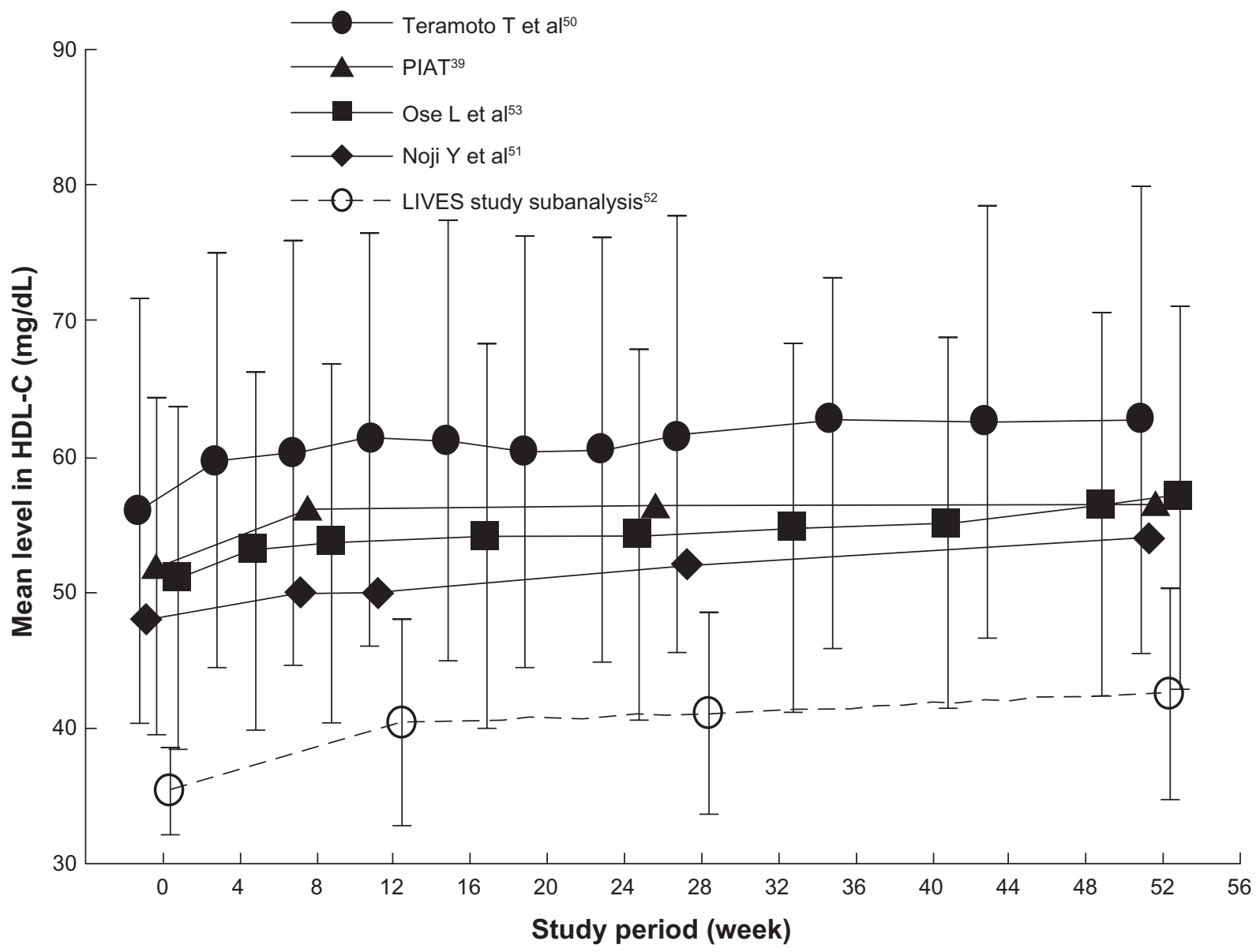

Figure I Time course of high-density lipoprotein cholesterol levels in long-term clinical studies of pitavastatin.

Note: Data are expressed as mean \pm standard deviation. Reference 52 is subanalysis for patients whose baseline high-density lipoprotein cholesterol was $<40 \mathrm{mg} / \mathrm{dL}$. 
this statin will establish its position as a useful drug for preventing and treating cardiovascular disease.

\section{Acknowledgment}

Kowa Company Ltd provided the information on the randomized active-controlled clinical research of pitavastatin already published but not found in the search result in PubMed.

\section{Disclosure}

The author reports no conflict of interest in this work.

\section{References}

1. Endo A, Kuroda M, Tanzawa K. Competitive inhibition of 3-hydroxy3-methylglutaryl coenzyme A reductase by ML-236 A and ML-236B fungal metabolites, having hypocholesterolemic activity. FEBS Lett. 1976;72(2):323-326.

2. Shepherd J, Cobbe SM, Ford I, et al. Prevention of coronary heart disease with pravastatin in men with hypercholesterolemia. West of Scotland Coronary Prevention Study Group. $N$ Engl J Med. 1995;333(20):1301-1307.

3. Downs JR, Clearfield M, Weis S, et al. Primary prevention of acute coronary events with lovastatin in men and women with average cholesterol levels: Results of AFCAPS/TexCAPS. Air Force/Texas Coronary Atherosclerosis Prevention Study. JAMA. 1998;279(20): 1615-1622.

4. Sacks FM, Pfeffer MA, Moye LA, et al. The effect of pravastatin on coronary events after myocardial infarction in patients with average cholesterol levels. Cholesterol and Recurrent Events Trial investigators. N Engl J Med. 1996;335(14):1001-1009.

5. Scandinavian Simvastatin Survival Study Group. Randomised trial of cholesterol lowering in 4444 patients with coronary heart disease; the Scandinavian Simvastatin Survival Study (4S). Lancet. 1994;344(8934):1383-1389.

6. Ridker PM, Danielson E, Fonseca FA, et al; JUPITER Study Group. Rosuvastatin to prevent vascular events in men and women with elevated C-reactive protein. N Engl J Med. 2008;359(21): 2195-2207.

7. McClure DL, Valuck RJ, Glanz M, Murphy JR, Hokanson JE. Statin and statin-fibrate use was significantly associated with increased myositis risk in a managed care population. $J$ Clin Epidemiol. 2007;60(8):812-818.

8. Sakaeda T, Fujino H, Komoto C, et al. Effects of acid and lactone forms of eight HMG-CoA reductase inhibitors on CYP-mediated metabolism and MDR1-mediated transport. Pharm Res. 2006;23(3):506-512.

9. Neuvonen PJ, Niemi M, Backman JT. Drug interactions with lipidlowering drugs: Mechanisms and clinical relevance. Clin Pharmacol Ther. 2006;80(6):565-581.

10. Kurihara Y, Douzono T, Kawakita K, Nagasaka Y. A large-scale, long-term, prospective post-marketing surveillance of pitavastatin (LIVALO ${ }^{\circledR}$ Tablet) - LIVALO Effectiveness and Safety (LIVES) Study. Jpn Pharmacol Ther. 2008;36(8):709-731.

11. Saito Y. Critical appraisal of the role of pitavastatin in treating dyslipidemias and achieving lipid goals. Vasc Health Risk Manag. 2009;5:921-936.

12. Jadhav SB, Jain GK. Statins and osteoporosis: New role for old drugs. J Pharm Pharmacol. 2006;58(1):3-18.

13. Kajinami K, Mabuchi H, Saito Y. NK-104: A novel synthetic HMG-CoA reductase inhibitor. Expert Opin Investig Drugs. 2000; 9(11):2653-2661.

14. McKenney JM. New cholesterol guidelines, new treatment challenges. Pharmacotherapy. 2002;22(7):853-863.
15. Nozue T, Yamamoto S, Tohyama S, et al. Kanagawa PTCA Conference Study Group. Treatment with statin on atheroma regression evaluated by intravascular ultrasound with Virtual Histology (TRUTH Study): Rationale and design. Circ J. 2009;73(2):352-355.

16. Miyauchi K, Kimura T, Morimoto T, et al. Japan assessment of pitavastatin and atorvastatin in acute coronary syndrome (JAPAN-ACS): Rationale and design. Circ J. 2006;70(12):1624-1628.

17. Rashid M, Tawara S, Fukumoto Y, Seto M, Yano K, Shimokawa H. Importance of Rac1 signaling pathway inhibition in the pleiotropic effects of HMG-CoA reductase inhibitors. Circ J. 2009;73(2):361-370.

18. Yokoyama T, Miyauchi K, Kurata T, Satoh H, Daida H. Inhibitory efficacy of pitavastatin on the early inflammatory response and neointimal thickening in a porcine coronary after stenting. Atherosclerosis. 2004;174(2):253-259.

19. Kokudai M, Inui N, Takeuchi K, Sakaeda T, Kagawa Y, Watanabe H. Effects of statins on the pharmacokinetics of midazolam in healthy volunteers. J Clin Pharmacol. 2009;49(5):568-573.

20. Di B, Su MX, Yu F, et al. Solid-phase extraction and liquid chromatography/tandem mass spectrometry assay for the determination of pitavastatin in human plasma and urine for application to Phase I clinical pharmacokinetic studies. J Chromatogr B Analyt Technol Biomed Life Sci. 2008;868(1-2):95-101.

21. Deng JW, Song IS, Shin HJ, et al. The effect of SLCO1B1*15 on the disposition of pravastatin and pitavastatin is substrate dependent: The contribution of transporting activity changes by SLCO1B1*15. Pharmacogenet Genomics. 2008;18(5):424-433.

22. Ando H, Tsuruoka S, Yanagihara $\mathrm{H}$, et al. Effects of grapefruit juice on the pharmacokinetics of pitavastatin and atorvastatin. $\mathrm{Br} J \mathrm{Clin}$ Pharmacol. 2005;60(5):494-497.

23. Chung JY, Cho JY, Yu KS, et al. Effect of OATP1B1 (SLCO1B1) variant alleles on the pharmacokinetics of pitavastatin in healthy volunteers. Clin Pharmacol Ther. 2005;78(4):342-350.

24. Sakamoto T, Kojima S, Ogawa H, et al; MUSASHI-AMI Investigators. Usefulness of hydrophilic vs lipophilic statins after acute myocardial infarction: Subanalysis of MUSASHI-AMI. Circ J. 2007;71(9):1348-353.

25. Ono K, Kawasaki M, Tanaka R, et al. Integrated backscatter and intima-media thickness of the thoracic aorta evaluated by transesophageal echocardiography in hypercholesterolemic patients: Effect of pitavastatin therapy. Ultrasound Med Biol. 2009;35(2):193-200.

26. Mizuguchi Y, Oishi Y, Miyoshi H, Iuchi A, Nagase N, Oki T. Impact of statin therapy on left ventricular function and carotid arterial stiffness in patients with hypercholesterolemia. Circ J. 2008;72(4): 538-544.

27. Sakabe K, Fukuda N, Fukuda Y, et al. Comparisons of short- and intermediate-term effects of pitavastatin versus atorvastatin on lipid profiles, fibrinolytic parameter, and endothelial function. Int J Cardiol. 2008;125(1):136-138.

28. Nakamura T, Sugaya T, Kawagoe Y, Suzuki T, Inoue T, Node K. Effect of pitavastatin on urinary liver-type fatty-acid-binding protein in patients with nondiabetic mild chronic kidney disease. Am J Nephrol. 2006;26(1):82-86.

29. Nakamura T, Sugaya T, Kawagoe Y, Ueda Y, Osada S, Koide H. Effect of pitavastatin on urinary liver-type fatty acid-binding protein levels in patients with early diabetic nephropathy. Diabetes Care. 2005;28(11):2728-2732.

30. Yoshida O, Kondo T, Kureishi-Bando Y, Sugiura, et al. Pitavastatin, an HMG-CoA reductase inhibitor, ameliorates endothelial function in chronic smokers. Circ J. 2010;74(1):195-202.

31. Miyashita Y, Endo K, Saiki A, et al. Effects of pitavastatin, a 3-hydroxy3-methylglutarylcoenzyme a reductase inhibitor, on cardio-ankle vascular index in type 2 diabetic patients. J Atheroscler Thromb. 2009; 16(5):539-545.

32. Nakamura T, Sato E, Fujiwara N, et al. Co-administration of ezetimibe enhances proteinuria-lowering effects of pitavastatin in chronic kidney disease patients partly via a cholesterol-independent manner. Pharmacol Res. 2010;61(1):58-61. 
33. Nomura S, Inami N, Shouzu A, et al. The effects of pitavastatin, eicosapentaenoic acid and combined therapy on platelet-derived microparticles and adiponectin in hyperlipidemic, diabetic patients. Platelets. 2009;20(1):16-22.

34. Nakamura T, Obata JE, Kitta Y, et al. Rapid stabilization of vulnerable carotid plaque within 1 month of pitavastatin treatment in patients with acute coronary syndrome. J Cardiovasc Pharmacol. 2008;51(4):365-371.

35. Saito Y, Yamada N, Teramoto T, et al. Clinical efficacy of pitavastatin, a new 3-hydroxy-3-methylglutaryl coenzyme A reductase inhibitor, in patients with hyperlipidemia. Dose-finding study using the doubleblind, three-group parallel comparison. Arzneimittelforschung. 2002;52(4):251-255.

36. Yoshitomi Y, Ishii T, Kaneki M, et al. Efficacy of a low dose of pitavastatin compared with atorvastatin in primary hyperlipidemia: Results of a 12-week, open label study. J Atheroscler Thromb. 2006;13(2):108-113.

37. Nozue T, Michishita I, Ito Y, Hirano T. Effects of statin on small dense low-density lipoprotein cholesterol and remnant-like particle cholesterol in heterozygous familial hypercholesterolemia. J Atheroscler Thromb. 2008;15(3):146-153.

38. Kawashiri MA, Nohara A, Tada H, et al. Comparison of effects of pitavastatin and atorvastatin on plasma coenzyme Q10 in heterozygous familial hypercholesterolemia: Results from a crossover study. Clin Pharmacol Ther. 2008;83(5):731-739.

39. Sasaki J, Ikeda Y, Kuribayashi T, et al. A 52-week, randomized, open-label, parallel-group comparison of the tolerability and effects of pitavastatin and atorvastatin on high-density lipoprotein cholesterol levels and glucose metabolism in Japanese patients with elevated levels of low-density lipoprotein cholesterol and glucose intolerance. Clin Ther. 2008;30(6):1089-1101.

40. Hiro T, Kimura T, Morimoto T, et al; JAPAN-ACS Investigators. Effect of intensive statin therapy on regression of coronary atherosclerosis in patients with acute coronary syndrome: A multicenter randomized trial evaluated by volumetric intravascular ultrasound using pitavastatin versus atorvastatin (JAPAN-ACS [Japan assessment of pitavastatin and atorvastatin in acute coronary syndrome] study). J Am Coll Cardiol. 2009;54(4):293-302.

41. Toi T, Taguchi I, Yoneda S, et al. Early effect of lipid-lowering therapy with pitavastatin on regression of coronary atherosclerotic plaque. Comparison with atorvastatin. Circ J. 2009;73(8):1466-1472.

42. Sansanayudh N, Wongwiwatthananukit S, Putwai P, DhummaUpakorn R. Comparative efficacy and safety of low-dose pitavastatin versus atorvastatin in patients with hypercholesterolemia. Ann Pharmacother. 2010;44(3):415-423.
43. Yokote K, Bujo H, Hanaoka H, et al. Multicenter collaborative randomized parallel group comparative study of pitavastatin and atorvastatin in Japanese hypercholesterolemic patients: Collaborative study on hypercholesterolemia drug intervention and their benefits for atherosclerosis prevention (CHIBA study). Atherosclerosis. 2008; 201(2):345-352.

44. Lee SH, Chung N, Kwan J, et al. Comparison of the efficacy and tolerability of pitavastatin and atorvastatin: An 8-week, multicenter, randomized, open-label, dose-titration study in Korean patients with hypercholesterolemia. Clin Ther. 2007;29(11):2365-2373.

45. Saito Y, Yamada N, Teramoto T, et al. A randomized, double-blind trial comparing the efficacy and safety of pitavastatin versus pravastatin in patients with primary hypercholesterolemia. Atherosclerosis. 2002;162(2):373-379.

46. Park S, Kang HJ, Rim SJ, et al. A randomized, open-label study to evaluate the efficacy and safety of pitavastatin compared with simvastatin in Korean patients with hypercholesterolemia. Clin Ther. 2005;27(7):1074-1082.

47. Ose L, Budinski D, Hounslow N, Arneson V. Comparison of pitavastatin with simvastatin in primary hypercholesterolaemia or combined dyslipidaemia. Curr Med Res Opin. 2009;25(11): 2755-2764.

48. Budinski D, Arneson V, Hounslow N, Gratsiansky N. Pitavastatin compared with atorvastatin in primary hypercholesterolemia or combined dyslipidemia. J Clin Lipidol. 2009;4(3):291-302.

49. Hounslow NJ. Efficacy and safety of pitavastatin compared to pravastatin in elderly patients with primary hypercholesterolemia or combined dyslipidemia. J Clin Lipidol. 2007;1(5):439.

50. Teramoto T, Saito Y, Yamada N, et al. Clinical safety and efficacy of NK-104 (pitavastatin), a new synthetic HMG-CoA reductase inhibitor, in the long-term treatment of hyperlipidemia - results of a multicenter long-term study. J Clin Therap Med. 2001;17(6):885-913. Japanese.

51. Noji Y, Higashikata T, Inazu A, et al; Hokuriku NK-104 Study Group. Long-term treatment with pitavastatin (NK-104), a new HMG-CoA reductase inhibitor, of patients with heterozygous familial hypercholesterolemia. Atherosclerosis. 2002;163(1):157-164.

52. Teramoto T, Shimano H, Yokote K, Urashima M. Effects of pitavastatin (LIVALO Tablet) on high density lipoprotein cholesterol (HDL-C) in hypercholesterolemia. J Atheroscler Thromb. 2009;16(5): 654-661.

53. Ose L, Budinski D, Hounslow N, Arneson V. Long-term treatment with pitavastatin is effective and well tolerated by patients with primary hypercholesterolemia or combined dyslipidemia. Atherosclerosis. 2010;210(1):202-208
Vascular Health and Risk Management

\section{Publish your work in this journal}

Vascular Health and Risk Management is an international, peerreviewed journal of therapeutics and risk management, focusing on concise rapid reporting of clinical studies on the processes involved in the maintenance of vascular health; the monitoring, prevention and treatment of vascular disease and its sequelae; and the involvement of

\section{Dovepress}

metabolic disorders, particularly diabetes. This journal is indexed on PubMed Central and MedLine. The manuscript management system is completely online and includes a very quick and fair peer-review system, which is all easy to use. Visit http://www.dovepress.com/ testimonials.php to read real quotes from published authors. 